\title{
Altitudinal Effects on Cytogenetic and Breeding of Panicum turgidum Forssk
}

\author{
S. A. Haroun* \\ Biology Department, College of Education, Tanta University, Kafr El Sheikh, Egypt \\ Accepted January 17, 2000
}

\begin{abstract}
Summary Cytogenetic and breeding studies were carried out on Panicum turgidum from Saudi Arabia (South west area). The materials were collected from 5 sites and grouped into two main groups according to altitude level. The first group include three sites of collection at see level, whereas the second one include two sites at altitude of $2600 \mathrm{~m}$ above see level.

Karyotype structure of the first group accessions shows high similarity recording $30 \mathrm{~m}+4 \mathrm{sm}+$ 2t. Karyotype measurements of this group recording $1.93 \mu \mathrm{m}$ mean length of chromosome and arm ratio of 1.15 with very low standard error reflect the low variation between chromosomes within the set. In contrast the structure of karyotype in the second group shows $26 \mathrm{~m}+6 \mathrm{sm}+4 \mathrm{t}$, with significant variation in chromosome length recording $2.26 \mu \mathrm{m}$ and arm ratio of 1.26 . This in addition to the high values of standard error reflect the variation among chromosomes in length and centromere position within the set.

Meiotic behavior of the first group accessions shows a low percentage of disturbed cells compared to that of the second group. In contrast to the second group, normal chromosome association was also observed in the first group accompanied with high level of chiasma frequency. Close relationship was noted between chiasma frequency and regular meiosis in form of chromosome association.

A strong relation was indirectly also noted between chiasma frequency and seed set either in self or open pollination conditions for the two groups. Similarity of karyotype, normal meiosis and high pollen fertility for the first group (see level altitude) pointed to high stability in genome structure assuming a high potential for self compatibility of the accessions. The second group ( $2600 \mathrm{~m}$ altitude level) shows low fertility associated very strongly with other parameters suggest the partial self-compatible habit at such rich vegetation area which increase the probability of crossing with relatively compatible grasses.
\end{abstract}

Genus Panicum of family Poaceae is of economic importance particularly in subarid zones, providing both low-grade annual cereals and perennial forage species. It is with perhaps over 500 species distributed throughout tropical, subtropical, warm arid regions of the world, and perhaps the largest grass genera.

Although many species of this genus have been subjected to modern criteria of cytogenetic in many sites around the world by many authors (Haroun et al. 1992, Haroun 1995, Zuloaga 1991, Rahman 1988), many others are still in need for such modern criteria especially those belonging to middle east area. Previous studies suggest a wide range of chromosome base number of 8, 9, 10 and sometimes 11 (Jauhar and Joshi 1965, De wet and Anderson 1956, Gould 1960, Chen and Hsu 1961). It seems that 9 is the common basic number with diploid, tetraploid and hexaploid levels.

In south west Saudi Arabia (area of study) this genus is represented by 4 species (Migahid 1978). Panicum turgidum the most common grass species of this group; grows vigorously at see level and at Abha site $(2600 \mathrm{~m}$ above see level). Following program of study the cytogenetics of grass family Poaceae, Panicum turgidum consider a good material not previously studied in this

* Present address: Biology Department, College of Science, King Khalid University, Abha, P.o.Box 157, Saudi Arabia. 
area. Modern criteria of cytogenetic and breeding system were investigated in the two levels of altitude.

\section{Materials and methods}

Materials of Panicum turgidum were collected from their natural habitats and herbarium vouchers were checked for names based on Migahid (1978), and Chaudhary (1989). Collections from 5 sites were grouped into two main groups. The first group represented see level altitude and included three main sites (along Makkah, Abha Road), whereas the second one represented the altitude of $2600 \mathrm{~m}$ above see level (Abha City, South West Saudi Arabia) and comprised 2 sites of collection.

For karyotype, excised root tips of germinated seeds (1-2 cm long) were pretreated in $0.4 \%$ colchicine for $3 \mathrm{~h}$, fixed in $1: 3 \mathrm{v} / \mathrm{v}$ acetic alcohol for $24 \mathrm{~h}$, hydrolysed in $1 \mathrm{~N} \mathrm{HCl}$ at $60^{\circ} \mathrm{C}$ for $8-$ $10 \mathrm{~min}$, and stained in feulgen reagent for $24 \mathrm{~h}$. Fully contracted metaphase cells (up to 5 of 3 to 6 plants for each accession) were used for karyotype study and measurements.

Meiotic behavior and pollen characteristics were studied using young panicles. Buds were fixed in freshly prepared 1:3 solution of acetic alcohol at suitable time. Anthers were collected from predihecient florets, macerated and squashed in $0.2 \%$ aceto-orceine. Meiotic cells were examined for chromosome association and chiasma frequency at diakinesis, metaphase I, and other stages for irregularities.

Pollen fertility was also assessed using the same technique and calculated as a percentage of stained pollen grains.

For seed set study; self and open conditions were compared by enclosing panicles before anthesis using paper bags. To estimate seed set at least 100 florets were examined for each of five individuals. For open pollination panicles dehiscing pollens were placed next to siblings and knocked to release pollen on a number of successive days (Haroun et al. 1992).

\section{Results and discussion}

\section{Mitotic study}

Of the 5 sites studied, accessions of Panicum turgidum investigated were tetraploid $(2 n=36)$, with basic number of 9 , which is the most common number among majority of species within this genus (Haroun et al. 1992, Jauhar and Joshi 1965). The previous reports on chromosome number of this species are $2 n=18$ recorded by Mulay et al. (1955), and $2 n=6 x=54$ by Ferchichi et al. (1994), based on $\mathrm{x}=9$ as basic number.

Accessions of the first group sites did not differ markedly in mean chromosome length $(\mu \mathrm{m})$, recording values of 1.96, 1.89 and 1.94 for sites 1,2 and 3 respectively (Table 1). Mean chromosome lengths of accessions in this group are very close and have very low standard error $( \pm 0.02)$. This to some extent reflect a degree of homogeneity among chromosome sets of these accessions.

Table 1. Number, total and mean length of chromosomes (chr.1.) and arm ratio (a.r.) for 5 accessions of $P$. turgidum accessions at 2 levels of altitude

\begin{tabular}{ccccc}
\hline \hline Site & 2n & Total chr.l. \pm S.E. & $\bar{x}$ chr.l. \pm S.E. & a.r. \pm S.E. \\
\hline 1 & 36 & $70.6 \pm 0.9$ & $1.96 \pm 0.08$ & $1.11 \pm 0.09$ \\
2 & 36 & $68.0 \pm 1.3$ & $1.89 \pm 0.04$ & $10.9 \pm 0.07$ \\
3 & 36 & $96.8 \pm 1.1$ & $1.94 \pm 0.07$ & $1.12 \pm 0.05$ \\
4 & 36 & $79.6 \pm 1.7$ & $2.32 \pm 0.11$ & $1.32 \pm 0.11$ \\
5 & 36 & $83.5 \pm 2.1$ & $2.32 \pm 0.11$ & $1.32 \pm 0.13$ \\
\hline
\end{tabular}


a

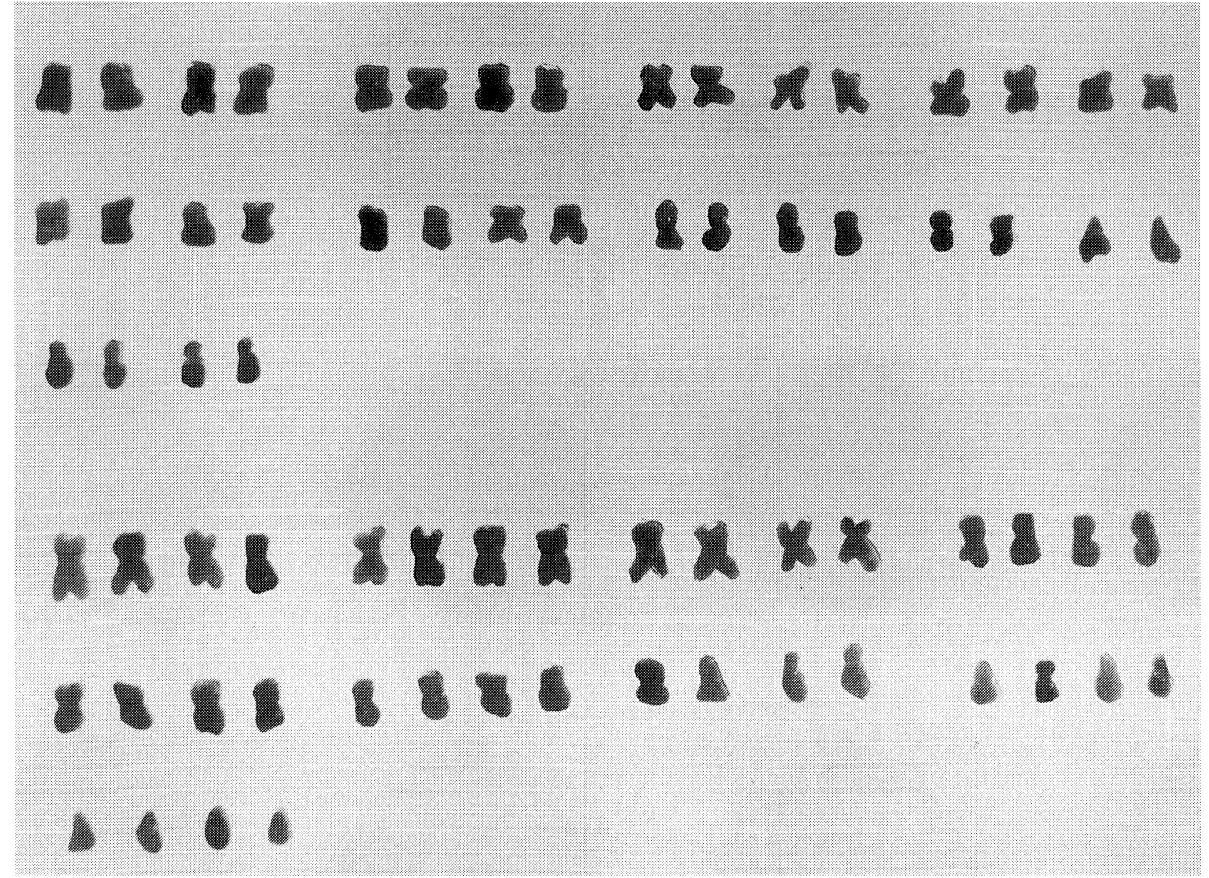

$\mathbf{a}^{\prime}$

HHH HH H H H Hi ii

$\mathbf{b}^{\prime}$

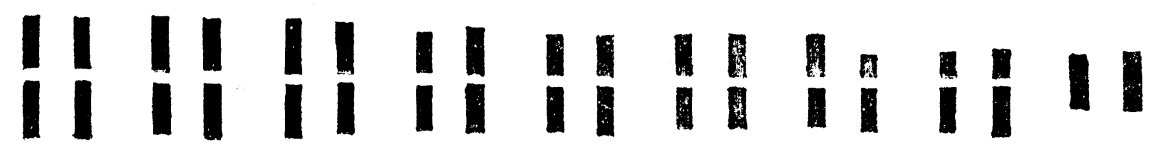

Fig. 1. Karyotype structure. a) First group accessions at sea level altitude $(30 m+4 s m+2 t)$. b) Second group accessions at level of $2600 \mathrm{~m}$ altitude $(26 \mathrm{~m}+6 \mathrm{sm}+4 \mathrm{t})$. $\mathrm{a}^{\prime}$ ) Idiogram of first group. $\left.\mathrm{b}^{\prime}\right)$ Idiogram of second group.

The value of arm ratio for these accessions ranged between 1.09-1.12 with mean value of $1.15 \pm 0.02$. The low standard error for both chromosome length and arm ratio to some extend prove the previous suggestion for karyotype structure and origin.

Based on length and centromere position karyotype structure of accessions in this group (Fig. 1a, $\mathrm{a}^{\prime}$ ) shows 15 pairs of metacentric, 2 pairs of submetacentric and one pair seems to be telocentric chromosomes $(30 m+4 s m+2 t)$. In spite of the presence of submetacentric and telocentric types, the majority of chromosomes in the set are similar and homologous in centromeres positions and the average lengths. Such measurements and karyotype structure suggest the autotetraploid origin of the species in the present study (Haroun 1997).

Karyotype structures of the two accessions of sites 4 and 5 (group 2), in contrast to the first group accessions; show more asymmetrical karyotype structure (Fig. 1b, $\mathrm{b}^{\prime}$ ) with significant variation among chromosomes within the set. Mean chromosome lengths of 2.21 and 2.32 were recorded for the two sites respectively with a mean value of 2.26 for them both which is highly comparable 
Table 2. Number of pollen mother cells (PMCs) examined, \% of iregular ones, chiasma frequency/bivalent (chia.freq.), $\%$ of pollen stainability (poll.stain.), and \% of seed set at open and self pollination (O.P., S.P.) for the 5 accession of $P$. turgidum at the 2 levels of altitude.

\begin{tabular}{|c|c|c|c|c|c|c|c|c|c|}
\hline \multirow{2}{*}{ Site } & \multirow{2}{*}{ No. PMCs } & \multirow{2}{*}{$\%$ irreg. PMCs } & \multicolumn{3}{|c|}{ chr. assoc. } & \multirow{2}{*}{ Chia. freq./biv. } & \multirow{2}{*}{$\%$ poll.stain. } & \multicolumn{2}{|c|}{$\%$ seed set } \\
\hline & & & uni. & bnv. & multi. & & & S.P. & O.P. \\
\hline 1 & 15 & 0.0 & 0 & 18 & - & 1.21 & 94.6 & 84.2 & 90.1 \\
\hline 2 & 17 & 5.1 & 0 & 14 & 2 & 1.17 & 59.1 & 85.2 & 93.7 \\
\hline 3 & 15 & 4.2 & 1 & 5 & - & 1.23 & 93.4 & 87.1 & 90.5 \\
\hline 4 & 14 & 6.7 & 2 & 15 & 1 & 1.01 & 85.1 & 69.3 & 77.1 \\
\hline 5 & 16 & 10.1 & 3 & 14 & 1 & 1.03 & 86.7 & 70.3 & 75.2 \\
\hline
\end{tabular}

Table 3. Mean values of chromosome length, arm ratio, $\%$ of irregularity, chiasma frequency, $\%$ of pollen stainability and seed set at self and open pollination condition for the 2 groups of $P$. turgidum

\begin{tabular}{|c|c|c|c|c|c|c|c|}
\hline \multirow{2}{*}{ Group } & \multirow{2}{*}{ chr.l. $\bar{x} \pm$ S.E. } & \multirow{2}{*}{ a.r. $\bar{x} \pm$ S.E. } & \multirow{2}{*}{$\%$ irreg. $\bar{x} \pm$ S.E. } & \multirow{2}{*}{ chia. freq. $\bar{x} \pm$ S.E. } & \multirow{2}{*}{$\%$ of stain. $\bar{x} \pm$ S.E. } & \multicolumn{2}{|c|}{$\overrightarrow{\mathrm{x}}$ seed set } \\
\hline & & & & & & S.P. & O.P. \\
\hline 1 & $1.93 \pm 0.02$ & $1.15 \pm 0.02$ & $3.1 \pm 1.28$ & $1.20 \pm 0.01$ & $94.4 \pm 0.41$ & $85.5 \pm 0.69$ & $91.4 \pm 0.93$ \\
\hline 2 & $2.26 \pm 0.04$ & $1.26 \pm 0.04$ & $8.4 \pm 1.20$ & $1.02 \pm 0.01$ & $85.9 \pm 0.56$ & $69.8 \pm 0.35$ & $76.2 \pm 0.67$ \\
\hline
\end{tabular}

with that of first group (1.93). A difference was noted in arm ratio of 1.26 compared with 1.15 for the first group.

Karyotype sttructure of the two accessions is very close showing 13 pairs of slightly long metacentric, 3 pairs of submetacentric and 2 pairs of telocentric chromosomes $(26 \mathrm{~m}+6 \mathrm{sm}+4 \mathrm{t})$. Compared with the first group, there is few difference in the number of metacentric, submetacentric and telocentric chromosomes and in their lengths. The high values of mean chromosome length, arm ratio and standard error, as well as the variation within chromosomes pointed to the hybrid structure of the karyotype.

To some extend such asymmetrical karyotype and variability within the set in this group is probably due to some chromosomal reorganization within the set, as one could predict that such organization would be accompanied by asymmetrical arm ratio. Based on measurements and variability in karyotype structure it could be assumed that group 2 accessions are under stress of structural hybridity as previously recorded by Haroun (1995) and Jauhar and Joshi (1965) for some species of Panicum.

\section{Male meiosis and seed set}

Accessions of the first group display a nearly regular meiosis with very low percentage of irregularities ranging between 0.0 to $5.1 \%$, with mean value of $3.1 \%$. Some of them seem to be a result of squash technique as disturbance, breaks and sometimes nondisjunction forms.

Chiasma frequency recorded for these accessions ranged between 1.17 and 1.23 with mean value of 1.20 , which is considered significantly high compared with other tetraploid species previously studied within this genus (Chen and Hsu 1961, Jauhar and Joshi 1965). Such high levels of chiasma frequency is probably a consequence of regular meiosis as normal association and the high level of bivalents recorded. The last parameter reflects the high degree of harmony between chromosomes in the set.

No dought the absence of univalents and low level of multivalents is positively affect chiasma frequency. As it is the site of crossover event where the genetic material exchange takes place, it 
could be expected that these accessions have active genes and possess functional gametes leading to increase fertility of pollen (recording 93.4 and 95.1 with mean value of 94.4 as in Tables 2, 3).

Not surprisingly, there is a very close relation between meiotic behavior and pollen stainability. The high level of chiasma frequency can be seen as evolutionary response to the challenge of fertility resulted from regular meiosis. Behavior of chromosomes in meiosis and their characteristics in mitosis pointed to genetic stability and autoploid nature of the species in this level of altitude.

Regarding seed set, a strong relationship was also found between pollen fertility and percentage of seed set either at open or self-pollination condition. As the percentage of self-seed set is higher nearly as that of open condition (recording $85.5 \%$ against $91.4 \%$ for the two conditions respectively), it could be assumed that these accessions are fully self-compatible (Haroun et al. 1992). This is also suggest that a major component of seed set is pollen fertility.

In contrast, the two accessions of the second group (altitude of $2600 \mathrm{~m}$ above see level), show a significantly high level of irregularities recording $8.4 \%$ against $3.1 \%$ for the first group (Table 3 ). However, irregularities observed were previously recorded in many species in this genus and some of related species. There is also a significant reduction in the percentage of pollen stainability recording $85.1 \%$ and $86.7 \%$ for the two accessions with mean value of $85.9 \%$ against mean value of $94.4 \%$ for the first group. This insures the strong relationship between meiotic behavior and pollen stainability.

Chromosome association in this group is significantly different from that of the first group, where many univalents (2-3) and low bivalents (13-15) were recorded. Multivalents observed were mostly in form of trivalents and few quadrivalents. No dought that low chiasma frequency of this group recording 1.02 against 1.20 for the first one which is highly affected by chromosome association (low bivalents and high univalents). In contrast low level of chiasma in this group could be considered as an evolutionary response to the challenge of infertility or partial fertility resulting from multivalents and chromosome association in alloploid accession.

Not suprisingly, abnormal meiosis and low chiasma frequency affect synaptic process and drastically reduce the frequency of crossing over. Consequently the gametes formed can not participate in pollination process and so increase the probability of open pollination system. This also insure the characteristics of self incompatibility of the accessions in this group. As there is no great difference in seed set at open and self pollination condition, this could also confirm the probability of cross pollination with some compatible grass or closely related species (Haroun 1995).

Following the results of meiotic behavior no dought that breeding system in this group is more likely to be partially self compatible, and the heterogeneity of genome structure play an important role regarding this system. It should not be ignored that reduced self pollination in this group also suggest that they may have intermediate partial self incompatibility from another unidentified parent.

As the 5 accessions were divided into two main groups based on altitude level, and found to differ markedly in all parameters investigated, it could be concluded that this factor (altitude level), plays an important role in cytogenetic stability of this species. Finally it should not be ignored that rich vegetation and high diversity of grass species at sites of group 2 accessions, to some extent increase the chance of cross pollination with these accessions. This seems to be the first step of genetic instability and the evolution of the species at this area.

\section{References}

Chaudhary, S. A. 1989. Grasses of Saudi Arabia. National Herberium, Ministry of Agriculture and Water. Kingdom of Saudi Arabia.

Chen, C. and Hsu, C. 1961. Cytogenetical studies on Taiwan grases I. Trib. Paniceae. Bot. Bull. Acad. Sci. Ser. 2: 101-110. 
De Wet, J. M. J. and Anderson, L. J. 1956. Chromosome numbers in transversal grasses. Cytologia 21: 1-10.

Ferchichi, A., Nabli, M. A. and Delay, J. 1994. Karyological investigation in the Poaceae from Tunisian steppes. ActaBotanica-Gallica 141(3): 327-341.

Gould, F. W. 1960. Chromosome number in southwestern grasses II. Amer. J. Bot. 47: 873-877.

Haroun, S. A. 1995. Cytological abnormality controls seed set in Panicum repens L. in Egypt. Cytologia 60: $347-351$.

- 1997. Cytogenetic studies on Seteria verticillata and Eragrostis egyptiaca from Egypt. Cytologia 62(2): 405-410.

-, Richards, A. J. and Macleod, R. D. 1992. Genetic relationship between some millets (Panicum). Linn. Soc. of London 13: 269-282.

Jauhar, P. P. and Joshi, A. B. 1965. Cytological studies in some species of Panicum. Cytologia 31(2): 153-159.

Migahid, A. M. 1978. Flora of Saudi Arabia. King Saud Univ. Publ., Saudi Arabia.

Mulay, B. N., Ramanathan, K. and Pounamma, M. 1955. Cytology of the grass Panicum turgidum Forssk. Proc. Ragasthan Acad. Sci. 5: 35-36.

Rahman, M. M. 1988. Taxonomic studies in the genus Panicum L. Ph.D. thesis. Univ. Edinb.

Zuloaga, F. O. 1991. Systematic of new world species of Panicum (Poaceae). An Integrated approach to the classification of the genus. 